Paper

\title{
Analysis of burst dynamics bound by potential with active areas
}

\author{
Koji Kurose ${ }^{1 a)}$, Yoshihiro Hayakawa ${ }^{2}$, Shigeo Sato ${ }^{1}$, \\ and Koji Nakajima ${ }^{1}$ \\ ${ }^{1}$ Laboratory for Brainware/Laboratory for Nanoelectronics and Spintronics, \\ Research Institute of Electrical Communication, Tohoku University \\ 2-1-1 Aoba Katahira, Sendai 980-8577, Japan \\ ${ }^{2}$ Sendai National College of Technology \\ 4-16-1 Aobaku Ayasi chuou, Sendai 989-3128, Japan \\ a)kurose@nakajima.riec.tohoku.ac.jp
}

Received February 23, 2011; Revised July 7, 2011; Published October 1, 2011

\begin{abstract}
Burst firing dynamics that are observed in many neuron models including the Hodgkin-Huxley model, are explained in terms of a motion of a quasi particle bound by potential. We are able to foresee the solution landscape with the curvature of the potential, and can design the wave form of the output to properly set active areas on the potential. In this paper, we apply this concept for a single Hindmarsh-Rose model and a coupled van der Pol oscillators. Therefore, we provide an understanding of the burst firings with spatiotemporal constructions of the potential and the active areas, and claim that the active areas cause the eigen-oscillations individually. Hence, we dispose the active areas on the potential properly and design the intended wave forms. Then, the global curvature of the potential function ensures that these oscillations do not diverge.
\end{abstract}

Key Words: Hindmarsh-Rose model, van der Pol oscillator, coupled oscillators, equation of motion, burst dynamics

\section{Introduction}

Many neuron models have been proposed through the years, including the Hodgkin-Huxley $(\mathrm{H}-\mathrm{H})$ model. They all have common properties, for example, generating spikes, burst firing, and so on. These models are nonlinear and have critical points where the behavior changes drastically depending on parameters. Hence, these behaviors, including burst oscillation, are discussed in terms of the stability of equilibrium points with the bifurcation theory in each model $[1,2]$. Furthermore, the behavior of coupled neuron systems is put in focus. Synchronization of nonlinear oscillators has been especially investigated and expected as an important role in artificial neural networks $[3,4]$.

In order to discuss the bursting dynamics globally, we have proposed the Inverse function Delayed (ID) model [5] and the Burst ID model of H-H type. The Burst ID model has characteristics of burst oscillation [8]. We think that the characteristics of burst oscillation provide an effective capacity for information processing of neurons, for example, robust signal propagation [6] and selective signal 
propagation among neurons [7].

We are able to explain the characteristics and various wave forms as a motion of "a quasi-particle" bound by the potential with the active areas. We are able to foresee the solution landscape with the curvature of the potential, and its dynamics are explained in relation to the disposition of active areas on the potential. This property can be applied to other neuron models including chaotic dynamics. We have already obtained the potential function and active areas for the Morris-Lecar model, the two dimensional Hindmarsh-Rose type model and so on [9-11].

In this paper, we discuss some bursting oscillations in terms of two kinds of modifications, and we claim that we can design wave forms of the bursting oscillations, and reveal the process the transition of the neuronal dynamics in detail. First, we apply our concept to a single bursting neuron model, and discuss some bursting dynamics with a motion equations, which have a third time derivative term and describe the motion of "a quasi-particle". Second, we analyze various bursting modes with spatiotemporal property of the potential functions of ordinary motion equations, which do not have a third time derivative term, and hence, describe the dynamics of a common particle. The analyses with ordinary motion equation help us to understand the motion of a quasi-particle described by the higher order differential equation.

As a model, we choose the Hindmarsh-Rose model [12] for the single bursting system. This model is similar to the Burst ID model in many ways, for instance, the characteristics of spiking, bursting and chaos. Meanwhile, we treat the van der Pol oscillator for the coupled system, because it is so simple that we can analyze its dynamics easily.

This paper is organized as follows. In Sec. 2, we introduce our technique, a concept of potential with active area, and discuss the stability of the equilibrium points and the proof of convergence or divergence of the solution. We apply our concept to the Hindmarsh-Rose model and discuss two type of the bursting dynamics in Sec. 3. In Sec. 4, we apply our concept to a coupled van der Pol oscillators, and we show that we are able to discuss the behavior based on our concept. Section 5 concludes this paper with a summary.

\section{Potential and active areas}

In this section, we introduce our concept, which is analysis with potential and active areas, and we give a detailed presentation of our concept.

Neuron models are typically expressed in the form of multidimensional nonlinear differential equations, and several models can be transformed into a higher differential equation with one variable as follows.

$$
\begin{aligned}
& \frac{d^{n} x}{d t^{n}}+b_{n-1}\left(x, \frac{d x}{d t}, \cdots, \frac{d^{n-1} x}{d t^{n-1}}\right) \frac{d^{n-1} x}{d t^{n-1}}+\cdots+b_{1}\left(x, \frac{d x}{d t}\right) \frac{d x}{d t} \\
& =F(x)=-\frac{\partial U(x)}{\partial x},
\end{aligned}
$$

where $U(x)$ is a kind of a potential function with some equilibrium points $x_{0}^{\prime} s$. When we determine the stability of the equilibria, considering the microscopic displacement $x_{0}+A \exp \lambda t$ around the equilibria, we obtain the characteristic equation,

$$
\lambda^{n}+b_{n-1}\left(x=x_{0}, \frac{d x}{d t}=0, \cdots, \frac{d^{n-1} x}{d t^{n-1}}=0\right) \lambda^{n-1}+\cdots+b_{1}\left(x=x_{0}, \frac{d x}{d t}=0\right) \frac{d x}{d t}+b_{0}\left(x=x_{0}\right)=0,
$$

where $n$ denotes the number of variables. According to Hurwitz's theorem we can analyze the stability at the vicinity of equilibria .

The equilibrium point $x_{0}$ is stable $(\mathrm{n}=3)$, if

$$
\begin{aligned}
& b_{0}\left(x_{0}\right)=\frac{\partial^{2} U\left(x=x_{0}\right)}{\partial x^{2}}>0, \\
& b_{i}\left(x_{0}\right)>0,(i=0,1,2) \\
& B_{1}\left(x_{0}\right)=b_{2}\left(x_{0}\right) b_{1}\left(x_{0}\right)-b_{0}\left(x_{0}\right)>0 .
\end{aligned}
$$


Equation (3) means that the curvature $b_{0}\left(x_{0}\right)$ of the equilibrium point of the potential function $U(x)$ is positive at $x_{0}$. In addition, $b_{0}(x)$ function determines the sign of the curvature of the potential function. When this function is positive for $x \rightarrow \pm \infty$, the potential function is a concave form, and it ensures that the solution will not diverge and be bounded. The motion equation describes the motion of a quasi-particle when $n \geq 3$.

An equilibrium point $x_{0}$ is unstable when $b_{1}\left(x_{0}\right)<0$ or $b_{2}\left(x_{0}\right)<0$ or $B_{1}\left(x_{0}\right)<0$ is satisfying, even if the global form of the potential function is positive. In other words, if there is no equilibrium point satisfying the requirement of Hurwitz's theorem and the global curvature is positive, it causes various oscillations, for example, a periodic limit cycle, burst oscillation and so on. $X_{b_{i}(x)<0}$ denotes the interval of $x$ where $b_{i}(x)<0(i=1,2)$ is satisfied. We refer to this area as a $b_{i}$-active area. The interval $X_{B_{1}(x)<0}$ where a $B_{1}(x)<0$ is referred to as a $B_{1}$-active area. A quasi-particle can not stay on the equilibrium point in these active areas, hence, we observe a oscillation of a quasi-particle around these equilibrium point. We consider that the active area give an eigen oscillating component to a quasi-particle [5].

Consequently, the dynamics of neuron models are expressed by a motion of a quasi-particle in the potential with active areas. However, it is not easy to clearly understand a motion of a quasi-particle described by the higher order motion equation.

Therefore, in the following sections, we discuss the dynamics through a higher order motion equation as the first step and provide an understanding of the bursting oscillation. There is a relation between a location of the active areas and a wave form of the output, hence, the location shows a qualitative nature of the burst oscillations.

In the second step, we analyze the dynamics through motion equations without a third time derivative terms, it means two subsystems with an implicitly time-dependent potential. The result we obtain provides a better understanding of the motion of a quasi-particle in the higher order differential system.

\section{Hindmarsh Rose type model}

In this section, we use the Hindmarsh-Rose model as a neuron model, which has bursting dynamics, and discuss the dynamics through our concept. We express that the active areas generate an eigenoscillation individually with one variable modification and the alteration between two bursting wave forms depends on the interval between the $b_{1}$-active area and the $b_{2}$-active area. This transition is expressed as a transformation of the potential function with two variables modification. As a result, we are able to design the form of bursting oscillations.

Hindmarsh-Rose model has three variables in its equation, hence, when we obtain one variable in its equation according to Sec. 2, the equation is the third order differential equation.

\subsection{Basic equations}

This model reads as follows

$$
\begin{aligned}
\tau_{x} \frac{d x}{d t} & =u-z-\epsilon\left\{\frac{1}{3} x^{3}-\alpha x^{2}+\left(\alpha^{2}-\beta\right) x\right\}+I_{e x t}, \\
\frac{d u}{d t} & =-u-d x^{2}+\theta, \\
\frac{d z}{d t} & =r\left\{s\left(x-Z_{0}\right)-z\right\},
\end{aligned}
$$

where $x, u$, and $z$ are variables, $\alpha, \beta, \epsilon, s, d$, and $Z_{0}$ are parameters, $I_{e x t}$ and $\theta$ denote a stimulate current and a threshold, respectively. We introduce the time constant $\tau_{x}$ on the output $x$, and $r$ is a parameter equivalent to a time constant.

\subsection{One variable modification}

Equations (6), (7), and (8) are transformed into a one variable equation expressed as

$$
\frac{d^{3} x}{d t^{3}}+b_{2}(x) \frac{d^{2} x}{d t^{2}}+b_{1}(x, \dot{x}) \frac{d x}{d t}=-\frac{\partial U(x)}{\partial x},
$$


where

$$
\begin{aligned}
& U(x)=\frac{r}{\tau_{x}}\left[\epsilon\left\{\frac{1}{12} x^{4}-\frac{1}{3} \alpha x^{3}+\frac{1}{2}\left(\alpha^{2}-\beta\right) x^{2}\right\}+\frac{1}{3} d x^{3}+\frac{1}{2} s x^{2}-\left(s Z_{0}+I_{\text {ext }}+\theta\right) x\right], \\
& b_{2}(x)=\frac{1}{\tau_{x}}\left\{\epsilon\left\{(x-\alpha)^{2}-\beta\right\}+\tau_{x}(r+1)\right\}, \\
& b_{1}(x, \dot{x})=\frac{1}{\tau_{x}}\left\{(r+1) \epsilon\left\{(x-\alpha)^{2}-\beta\right\}+2 d x+r\left(s+\tau_{x}\right)+2 \epsilon(x-\alpha) \dot{x}\right\} .
\end{aligned}
$$

When the model is expressed in the form of a differential equation with one variable $x$, the system has three active areas $X_{b_{1}(x)<0}, X_{b_{2}(x)<0}$, and $X_{B_{1}(x)<0}$ according to Sec. 2, and

$$
\begin{aligned}
& B_{1}(x, \dot{x}=0)=b_{2}(x) b_{1}(x, \dot{x}=0)-b_{0}(x), \\
& b_{0}(x)=\frac{\partial^{2} U(x)}{\partial x^{2}}=\frac{r}{\tau_{x}}\left[\epsilon\left\{x^{2}-2 \alpha x+\left(\alpha^{2}-\beta\right)\right\}+2 d x+s\right] .
\end{aligned}
$$

The potential function $U(x)$ Eq. (10) is a quartic function, therefore, it ensures that the solutions do not diverge if $\frac{r \epsilon}{\tau_{x}}>0$ and active areas are localized.

Supposing that the condition, $r<<\tau_{x}$, is satisfied, the $b_{2}$-active area $X_{b_{2}(x)<0}$ is

$$
X_{b_{2}(x)<0} \equiv\left[\alpha-\sqrt{\beta-\tau_{x} / \epsilon}, \alpha+\sqrt{\beta-\tau_{x} / \epsilon}\right],
$$

and the $B_{1}$-active area overlaps the others almost completely because $b_{0}(x)$ take the tiny value, hence, we ignore the $B_{1}$-active area in this section.

Next, I give a simulation result that the oscillations with an proper frequency are occurred around the b- 1 and the b-2 active areas, and the oscillations has an amplitude closely connected with the interval between the $b_{1}$ and the $b_{2}$-active area. Figure 1 shows time series of the output $x(t)$ and the potential function $U(x)$ when we perform this numerical calculation under the initial conditions $x=1, u=z=0$. The potential level is depicted as a contour line map. Yellow and blue areas denote the $b_{1}$ and the $b_{2}$-active areas, respectively. The active areas, the potential, and its equilibrium point do not change with time.

The potential function has a single well form and one equilibrium point. The equilibrium point is in the $b_{1}$-active area and destabilized, hence, the output shows continuous oscillations.

If $\tau_{x}=1$, the Hindmarsh-Rose system generates a typical bursting output, which has some spikes without undershoot shown in Fig. 1(a). The amplitude of the firing oscillation between each resting state tapers off with increasing $\tau_{x}$ (Fig. 1(b)), hence, the bursting output of Fig. 1(b) is referred to as a tapered bursting [1]. The oscillation around the equilibrium point is slow. A value $\tau_{x} \approx 1.21$ is found to define this transition of wave forms.
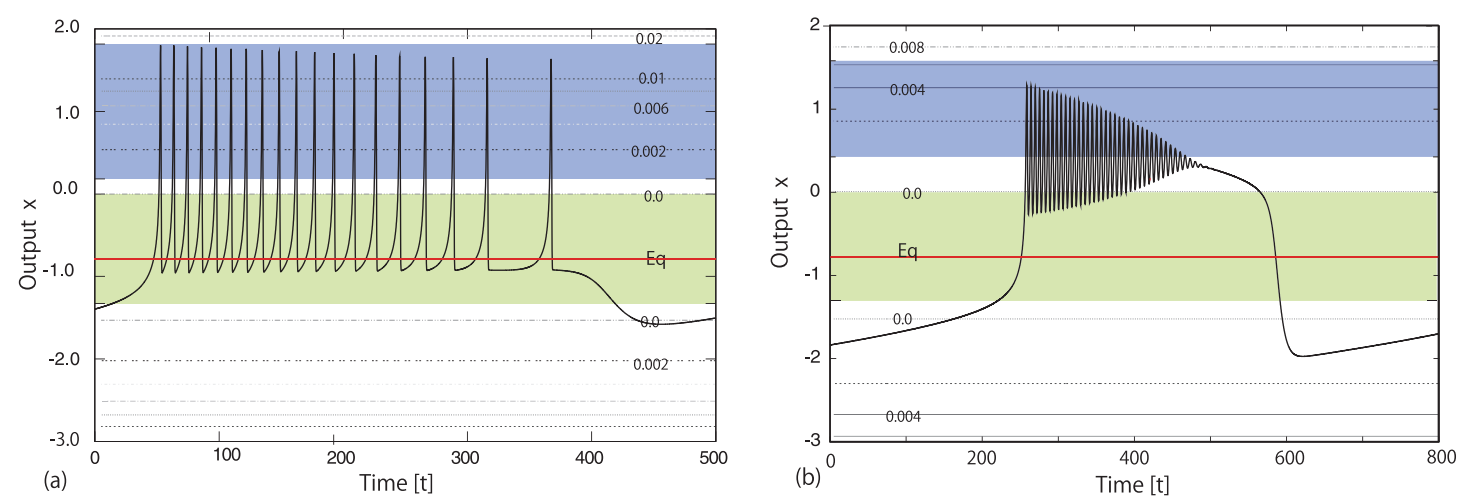

Fig. 1. Time series of the output $\mathrm{x}(\mathrm{t})$ and potential function $U(x)$ for the Hindmarsh-Rose model, when $\alpha=1.0, \beta=1.0, \epsilon=3.0, I_{\text {ext }}=3.0, d=5.0$, $\theta=1.0, s=4.0, Z_{0}=-1.6, r=0.001$, (a) $\tau_{x}=1.0$, and (b) $\tau_{x}=2.0$. Black lines, red lines, yellow areas and blue areas denote the outputs, the equilibrium points, the $b_{1}$-active areas and the $b_{2}$-active areas, respectively. 
Figure 1(b) shows that a quasi-particle stops and runs up the wall of the potential around the $b_{1}$ active area and this movement represents the fast oscillations in the exciting state. These dynamics cannot be explained with conventional particle motions. This uncommonsensical oscillation occurs around the $b_{2}$-active area, hence, we are able to consider that this active area gives the fast oscillating component to a quasi-particle.

The width of the interval between the $b_{1}$-active area and the $b_{2}$-active area increases with increasing the parameter $\tau_{x}$. The difference of the amplitudes between Figs. 1(a) and (b) depends on an interval that is a non-active area between the $b_{1}$-active area and the $b_{2}$-active area. A quasi particle loses its energy in the interval. Therefore, the wider interval in (b) than (a) causes the tapering of the amplitude of fast oscillation, because a quasi particle loses more energy. Moreover, a quiescent state is expressed as a motion of a quasi particle that drops down toward the equilibrium point. After that, it runs up on the potential wall without a stop because of destabilization by the $b_{1}$-active area, and it moves in the $b_{2}$-active area with quick oscillation. The amplitude tapering in the non-active area and the movement with large amplitude around the unstable equilibrium point are not able to be explained by the bifurcation theory.

\subsection{Two variables modification}

We obtained the qualitative understanding of the busting oscillations in the previous subsection. In this subsection, we investigate the oscillation phenomena around the $b_{1}$-active area and the $b_{2}$-active areas and a mechanism of the wave form around the parameter $\tau_{x}=1.21$. The drastic change is caused by a manner of a transition of the potential function with time. We modify the basic equation to discuss the dynamics with a particle motion in a time dependent potential. We can analyze in detail the characteristics of the large-amplitude oscillations in the following modification.

Therefore, we eliminate the variable $u$ and transform Eqs. (6), (7), and (8) into the following equations.

$$
\begin{aligned}
\frac{d^{2} x}{d t^{2}}+\eta(x) \frac{d x}{d t} & =F(x, z)=-\frac{\partial U(x, z)}{\partial x}, \\
\frac{d z}{d t} & =r\left\{s\left(x-Z_{0}\right)-z\right\}
\end{aligned}
$$

where $\eta(x), F(x, z)$, and $U(x, z)$ are expressed as

$$
\begin{aligned}
\eta(x) & =\frac{1}{\tau_{x}} \epsilon\left\{(x-\alpha)^{2}-\beta\right\}+1, \\
F(x, z) & =-\frac{1}{\tau_{x}}\left\{\epsilon\left(\frac{1}{3} x^{3}-\alpha x^{2}+\left(\alpha^{2}-\beta\right) x\right)+d x^{2}+r s\left(x-Z_{0}\right)-\left(I_{e x t}+\theta+(r-1) z\right)\right\},
\end{aligned}
$$

and $\left.U(x, z)=\frac{1}{\tau_{x}}\left\{\epsilon\left\{\frac{1}{12} x^{4}-\frac{1}{3} \alpha x^{3}+\frac{1}{2}\left(\alpha^{2}-\beta\right) x^{2}\right)\right\}+\frac{1}{2} r s\left(x^{2}-2 Z_{0} x\right)-\left(I_{\text {ext }}+\theta+(r-1) z\right) x\right\}$,

respectively. The potential function $U(x, z)$ is a quartic function and includes two variables $x$ and $z$. There is an active area $X_{\eta(x)<0}$ calculated by Eq. (18) where the viscosity resistance is negative,

$$
X_{\eta(x)<0} \equiv\left[\alpha-\sqrt{\beta-\tau_{x} / \epsilon}, \alpha+\sqrt{\beta-\tau_{x} / \epsilon}\right] .
$$

In this modification, the equation of motion $x$ consists of an inertial term $\ddot{x}$, a viscosity resistance term $\eta(x) \dot{x}$ and a force from the potential $F(x, z)$, hence, we are able to discuss the dynamics of variable $x$ described with ordinal motion equation in general way.

The variable $z$ chases after the output $x$ and oscillates slowly because of $r<<\tau_{x}$, and hence, the potential function $U(x, z)$ is forced to oscillate and gradually changes its shape depending on variable $z$.

That is, the burst dynamics for Hindmarsh-Rose model is expressed as a Fast-Slow coupled system in this modification. 


\subsubsection{Bursting for $\tau_{x}=1$}

Figures 2 and 3 show a bird's eyes view and a contour map of time series of the potential function $U(x, z)$ Eq. (20), when $\tau_{x}=1$ (Fig. 1(a)). This bursting pattern is typically observed in many neuron models, and the dynamics are able to be explained in terms of a motion of a particle bound by a double well potential. The bursting mechanism is explained as follows.

When the particle is tumbling down to the stable equilibrium point without an active area, the output shows a resting state. Then the variable $z$ is chasing after $x$ and the potential leans in a positive direction, hence, the stable and unstable equilibrium points of the potential disappear at point (a) in

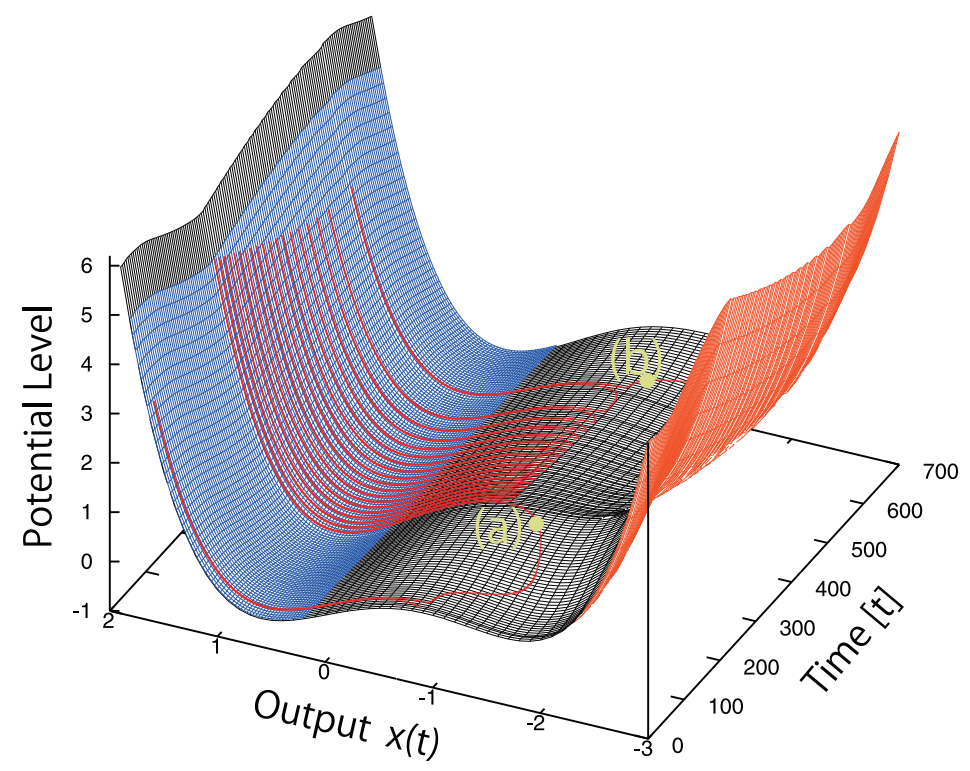

Fig. 2. Time series of bird's eye view with $\tau_{x}=1$. Blue area denotes the $\eta$-active area.

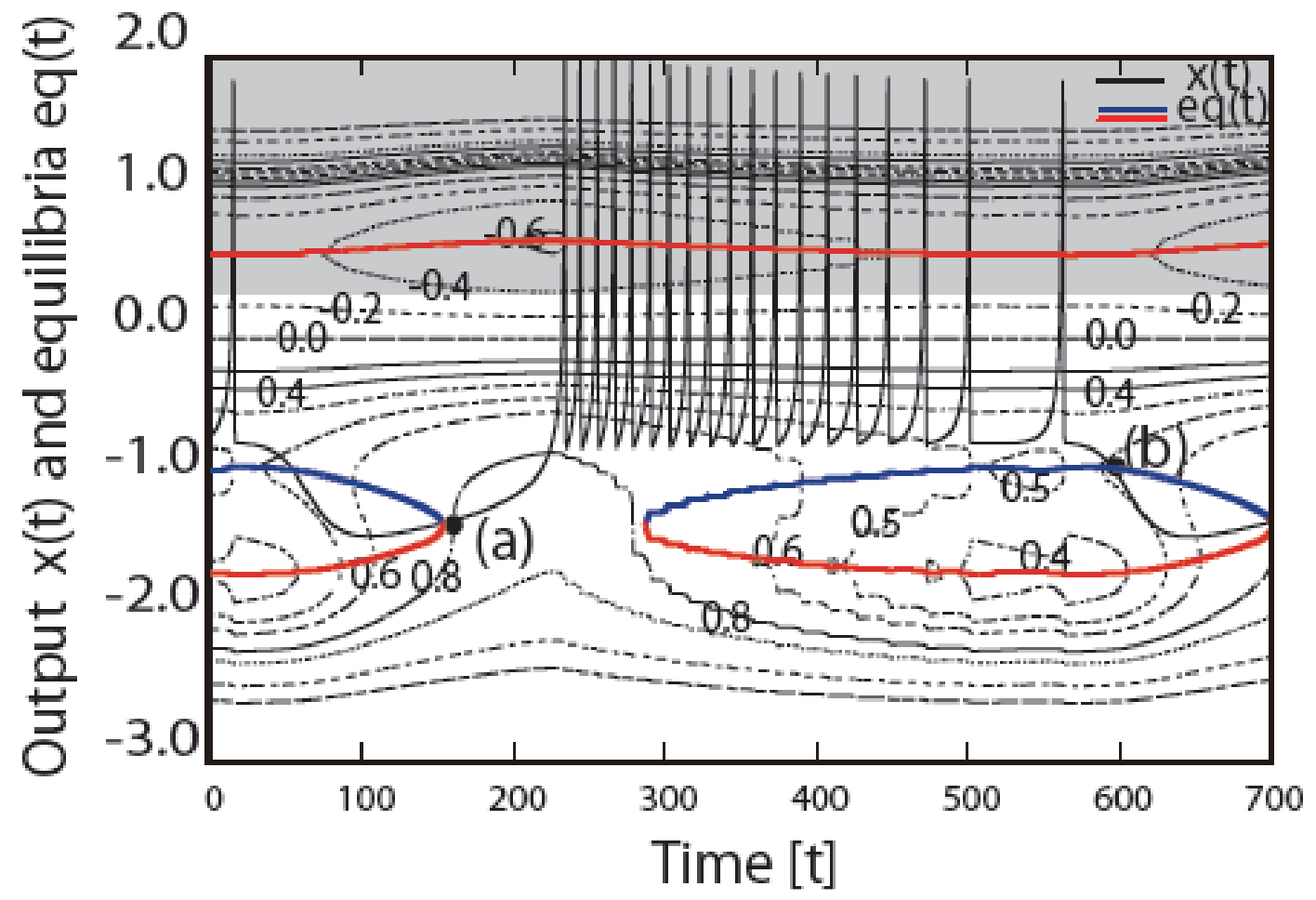

Fig. 3. Time series of the contour map of the potential with $\tau_{x}=1$. Black line, red lines, blue lines and gray area denote the output, trajectories of the equilibria with a positive curvature, trajectories of the equilibria with a negative curvature, and the $\eta$-active area, respectively. 
the Figs. 2 and 3. At this time, the potential becomes a single well potential. The particle tumbles down and moves into another equilibrium point. These transitions of the potential function $U(x, z)$ and movement of a particle show the neuronal ignition sequence.

The equilibrium point is destabilized by the $\eta$-active area $X_{\eta(x)<0}$ denoted by the blue zone in Fig. 2, and the particle oscillates in the valley around this equilibrium point. The firing oscillation in the exciting state is explained in this way.

The variable $z$ is moving to a positive direction and the potential leans in the reverse direction. After that, the potential becomes a double well form again, and the particle tumbles down to the stable equilibrium point after point (b) in Figs. 2 and 3. In this way, the output shows the resting state again.

\subsubsection{Tapered bursting for $\tau_{x}=2$}

When $\tau_{x}=2$, the output shows the oscillation with the tapered amplitude shown in the previous subsection. In this subsection, the mechanism is revealed with the Fast-slow system.

The bursting dynamics transfer from the resting state to the exciting state at point (a) in Figs. 4 and 5 in a similar way to the burst type for $\tau_{x}=1$. Then, the bursting oscillation occurs and the potential function turns back to the double well form as shown in Fig. 5. At this time, the particle oscillates on the vicinity of the equilibrium point in the $\eta$-active area. After that, a quasi-particle stops on the equilibrium point when the top equilibrium point in Fig. 5 moves out from the $\eta$-active area. In the next stage, the potential leans in the negative direction with increasing $z$, hence, the top stable equilibrium point disappears at point (b) in Fig. 5.

These potential transitions want the $\eta$-active area to be situated on the upper side of all equilibria. Therefore, this bursting pattern is observed, when the $\eta$-active area is situated further from the equilibrium point than any bursting pattern.

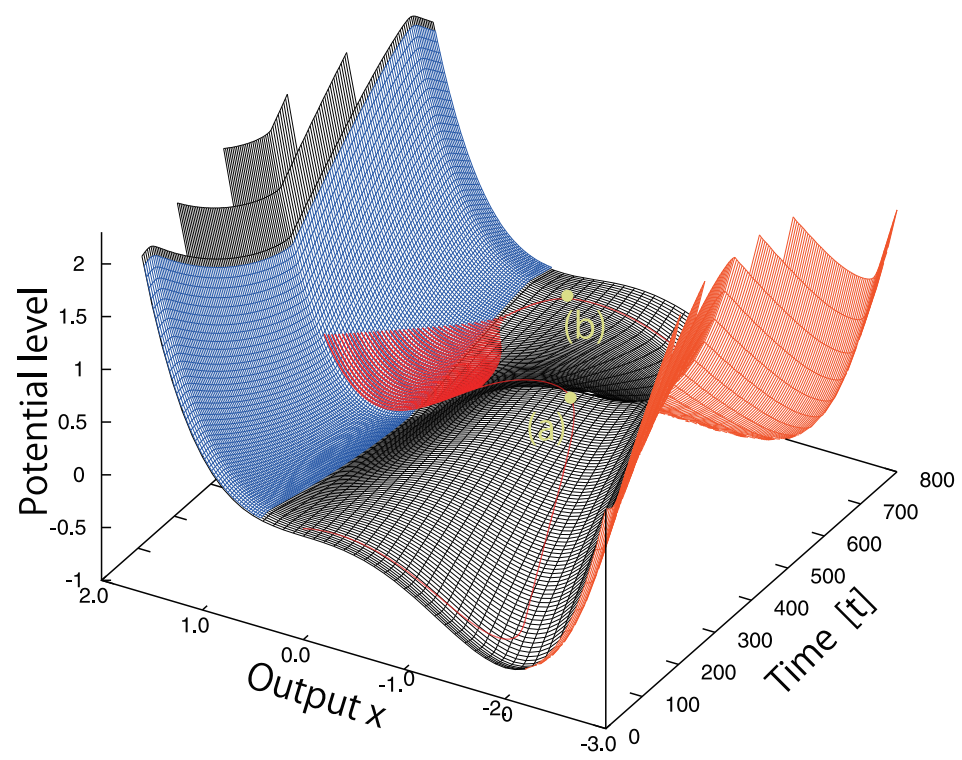

Fig. 4. Bird's eye view, when tapered bursting occurs with $\tau_{x}=2$.

Figure 6 shows the orbit of variable $z$, the motion equations are expressed by Eqs. (22) and (23),

$$
\begin{aligned}
& \frac{d z}{d t}=-\frac{\partial U(z, x)}{\partial z} \\
& U(z, x)=-r\left\{s\left(x-Z_{0}\right) z-\frac{1}{2} z^{2}\right\}
\end{aligned}
$$

This variable is not able to oscillate autonomously, and drifts in the potential $U(z, x)$.

We summarize the results so far. In one variable modification, the slow and fast oscillations of the bursting is observed around the $b_{1}$ and $b_{2}$-active areas, respectively. These oscillations are explained in two variable modifications as follows. 


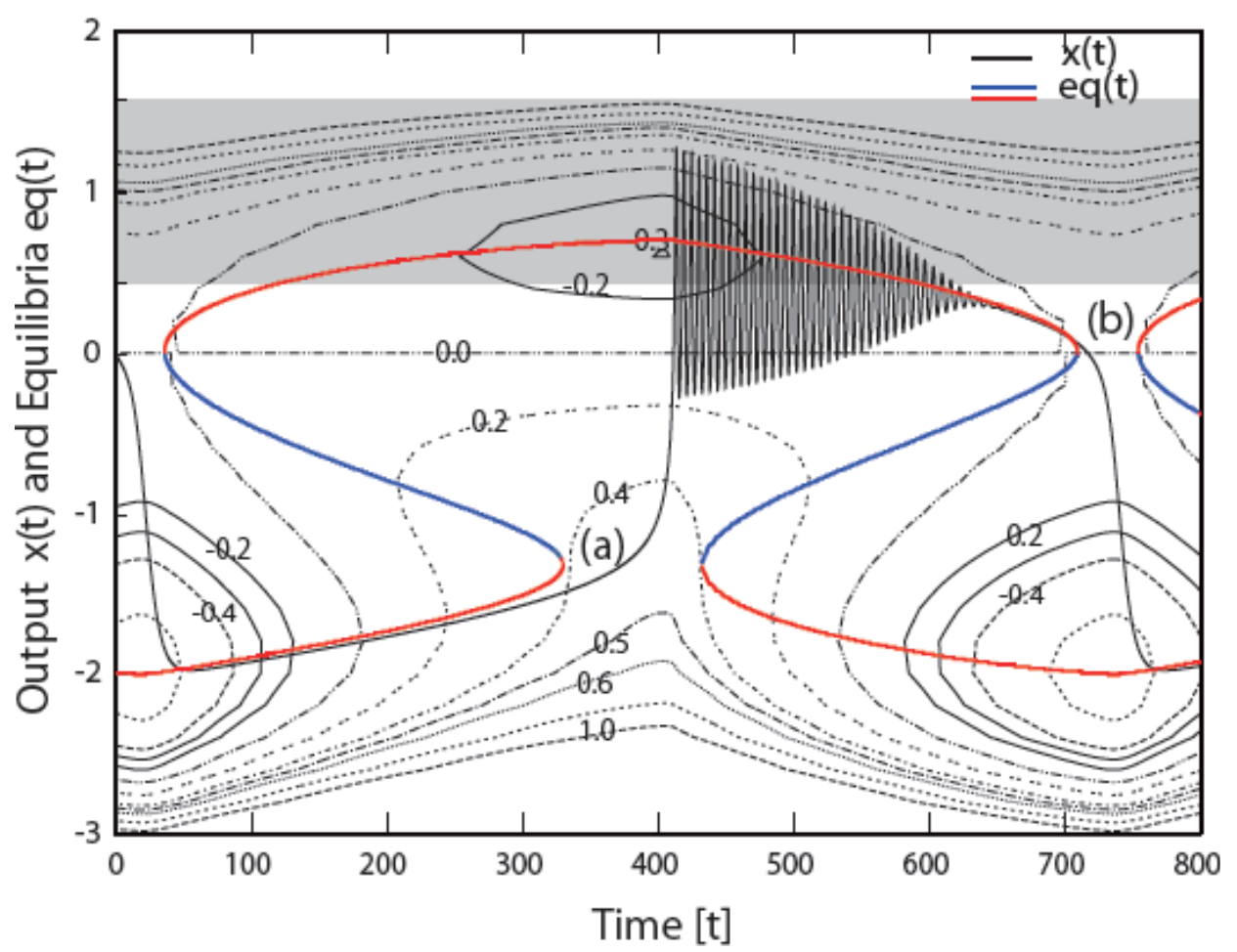

Fig. 5. Time series of the contour map of the potential and the output, with $\tau_{x}=2$. Black line, red lines, blue lines and gray area denote the output, trajectories of the equilibria with a positive curvature, trajectories of the equilibrium point with a negative curvature, and the $\eta$-active area, respectively.

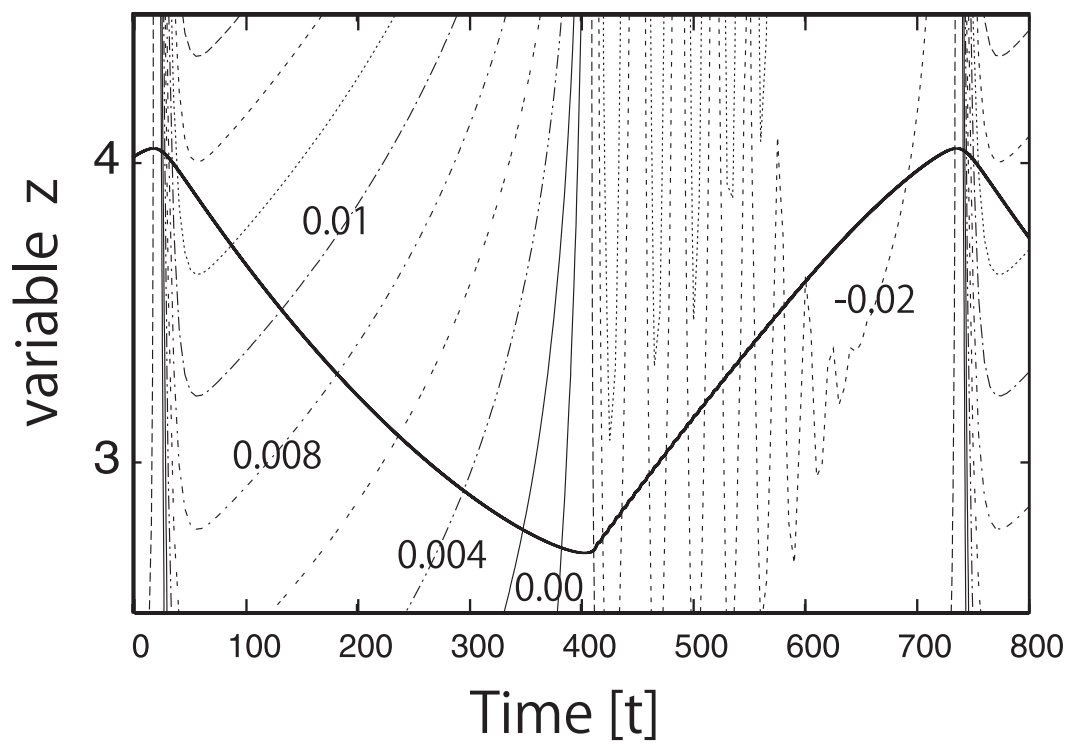

Fig. 6. Contour map for variable $z$ with $\tau_{x}=2$.

The movement of a particle by transformation of the potential function $U(x, z)$ corresponds to the oscillation of a quasi-particle around the equilibrium point destabilized by the $b_{1}$-active area in the previous subsections. It means that the characteristics of the $b_{1}$-active area is not explained as an energy injection part, but in relation to the motion of a particle that is forced to move by the transformation of the potential function $U(x, z)$, and this area dominates the slow dynamics of the burst oscillation.

The $b_{2}$-active area corresponds to the $\eta$-active area defined by Eq. (21). The width of $b_{2}$-active area calculated from Eq. (11) and the amplitude of the fast oscillation becomes narrower with increasing $\tau_{x}$. That is, the firing oscillations that are the same oscillations around the $\eta$-active area are occurred 
around the $b_{2}$-active area. These oscillations must not diverge, because the potential function has a concave form globally.

The one variable modification provides qualitative understanding, as the location of the $b_{2}$-active areas has close relation to the wave forms of the burst oscillations. The two variables modification gives us an understanding of some details about the differences of wave forms.

\section{4. van der Pol oscillator}

In this section, we apply our technique mentioned in the previous section to the coupled system as preliminary steps for the networked applications. We choice a van der Pol oscillator as a model, which has the simple autonomous oscillating dynamics. Two oscillators are coupled with a linear coupling, and the burst dynamics with spike undershoot is achieved. We discuss the burst dynamics for coupled van der Pol oscillators based on our concept of potential with active areas.

\subsection{Basic equations}

Generally the single van der Pol model is expressed with the following ordinary motion equation,

$$
\frac{d^{2} x}{d t^{2}}-q\left(1-x^{2}\right) \frac{d x}{d t}=-x .
$$

We consider the following equation of van der Pol type, because we can set the position and width of the active area arbitrarily considering the external input and time-delay,

$$
\frac{d^{2} x}{d t^{2}}+\frac{\epsilon}{\tau}\left\{(x-\alpha)^{2}-\beta\right\} \frac{d x}{d t}=\frac{1}{\tau}(W x+\theta)=-\frac{\partial U(x)}{\partial x},
$$

where $x, u, \theta$, and $\tau$ are the output of the unit, the internal state, the external input, and the time constant, respectively, and $\alpha, \beta, \epsilon$, and $W$ are control parameters. $U(x)$ is a potential function, described as

$$
U(x)=-\frac{1}{\tau}\left(\frac{1}{2} W x^{2}+\theta x\right) .
$$

The potential function gives the sign of the curvature of potential and one equilibrium point $x_{0}$,

$$
\begin{aligned}
& b_{0}=\frac{\partial^{2} U(x)}{\partial x^{2}}=-\frac{W}{\tau}, \\
& x_{0}=-\frac{\theta}{W} .
\end{aligned}
$$

The parameter $\tau$ is a time constant, hence, the value is always positive. If $W>0$, the potential function becomes a convex function, and we obtain a divergence solution because of Eq. (27). In contrast, the potential is a concave function if $W<0$.

When the equilibrium point is located within an active area, the output reveals continuous oscillation. This system expressed by Eq. (25) has one active area $b_{1}$-active area. The $b_{1}$-active area is the interval of $x$ where

$$
b_{1}(x)=\frac{\epsilon}{\tau}\left\{(x-\alpha)^{2}-\beta\right\}<0,
$$

and the $b_{1}$-active area $X_{b_{1}(x)<0}$ is defined with

$$
X_{b_{1}(x)<0} \equiv[\alpha-\sqrt{\beta}, \alpha+\sqrt{\beta}],
$$

where $\beta>0 . \alpha$ and $\beta$ denote the center of $b_{1}$-active area and its width $2 \sqrt{\beta}$, respectively. When we assign Eq. (28) to Eq. (29), we obtain the condition for oscillation requiring $W<0$. Figure 7 shows an example of the condition. 


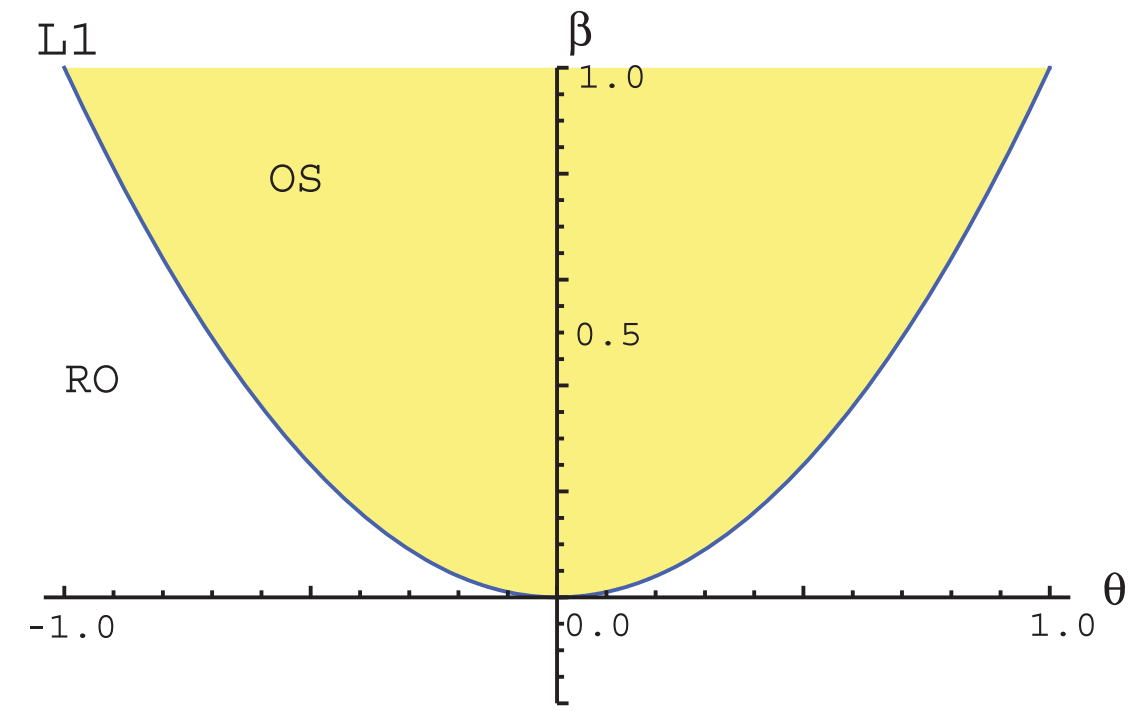

Fig. 7. Diagram of the output of the van der Pol system, RO and OS denote resting state and oscillation, respectively. The equilibrium point is within the $b_{1}$-active area above $\mathrm{L} 1$, when $\alpha=0.0, \epsilon=1.0$ and $W=-1$.

\subsection{Burst oscillation for coupled van der Pol oscillators}

We couple two units together described by Eq. (25). The coupling and self-connection strengths are $\omega_{i j}=\omega_{j i}=\omega$ and $W_{x}=W_{y}=W$, respectively, and $\alpha_{x, y}=0$.

There are two units $x$ and $y$ expressed with the following equations,

$$
\begin{gathered}
\tau_{x} \frac{d^{2} x}{d t^{2}}+\epsilon_{x}\left\{x^{2}-\beta_{x}\right\} \frac{d x}{d t}=W x+\omega y+\theta_{x}, \\
\tau_{y} \frac{d^{2} y}{d t^{2}}+\epsilon_{y}\left\{y^{2}-\beta_{y}\right\} \frac{d y}{d t}=W y+\omega x+\theta_{y},
\end{gathered}
$$

$x$ and $y$ denote the outputs of each unit. We transform Eqs. (31) and (32) into one variable equation according to Sec. II,

$$
\frac{d^{4} x}{d t^{4}}+b_{3}(x) \frac{d^{3} x}{d t^{3}}+b_{2}(x, \dot{x}, \ddot{x}) \frac{d^{2} x}{d t^{2}}+b_{1}(x, \dot{x}) \frac{d x}{d t}=-\frac{\partial U(x)}{\partial x},
$$

and the potential function is defined by

$$
U(x)=\frac{1}{\tau_{x} \tau_{y}}\left\{\frac{1}{2}\left(W^{2}-\omega^{2}\right) x^{2}+\left(W \theta_{x}-\omega \theta_{y}\right) x\right\},
$$

The potential function has a quadratic function. Equation (35) gives a concave form to the potential function if $|W|>|\omega|$. Therefore, this condition ensures that the oscillations generated in the potential do not diverge.

The active areas are defined as the following equations are satisfied,

$$
\begin{aligned}
& b_{1}(x)=-\frac{W}{\tau_{x} \tau_{y}} \eta(x)-\frac{W}{\tau_{x} \tau_{y}} \zeta(x)<0, \\
& b_{2}(x)=-\frac{W}{\tau_{x}}-\frac{W}{\tau_{y}}+\frac{1}{\tau_{x} \tau_{y}} \eta(x) \zeta(x)<0, \\
& b_{3}(x)=\frac{1}{\tau_{x}} \eta(x)+\frac{1}{\tau_{y}} \zeta(x)<0, \\
& B_{1}(x)=b_{3}(x) b_{2}(x)-b_{1}(x)<0, \\
& B_{2}(x)=B_{1}(x) b_{1}(x)-b_{3}^{2}(x) b_{0}(x)<0,
\end{aligned}
$$

where 


$$
\begin{aligned}
\eta(x)= & \epsilon_{x}\left(x^{2}-\beta_{x}\right), \\
\zeta(x)= & \epsilon_{y}\left\{\left(\frac{W x+\theta_{x}}{\omega}\right)^{2}-\beta_{y}\right\}, \\
b_{0}(x)= & \frac{\partial^{2} U(x)}{\partial x^{2}}, \\
& =\frac{1}{\tau_{x} \tau_{y}}\left(W^{2}-\omega^{2}\right) .
\end{aligned}
$$

The dynamics of unit $x$ are expressed as a motion of a quasi-particle in the single well potential with five active areas.

\subsection{Oscillations dominated by active areas}

In this subsection, we shows that the transition from simple slow oscillations to the bursting oscillation is caused by the damping around the equilibrium point located at the center of the quiescent state.

Figures $8(\mathrm{a}),(\mathrm{b})$, and (c) show the outputs $x(t)$, the potential function $U(x)$, and the active areas under a condition of $\tau_{x}<<\tau_{y}$, when the external input $\theta_{x}$ to unit $x$ has different values in each figures. The potential level is depicted as a contour line map, and red line denotes its equilibrium point. Both do not change with time. The equilibrium point is in the active areas expressed as gray, yellow and blue areas.

We perform the numerical simulation under the initial conditions $x=1, \dot{x}=0, y=1, \dot{y}=0$ with $\tau_{y}=100 \tau_{x}$, and Fig. 8 shows the outputs when it has passed enough time to make the system become a periodic steady state.

We present the slow simple oscillation, the spike pulse, and the burst oscillation with undershoot, which has four spikes per burst, in Figs. 8(a), (b), and (c), respectively. We find that the oscillations in Fig. 8 have more spikes with increasing the external input $\theta_{x}$. There are five active areas $b_{1}(x)<0$,

(a)
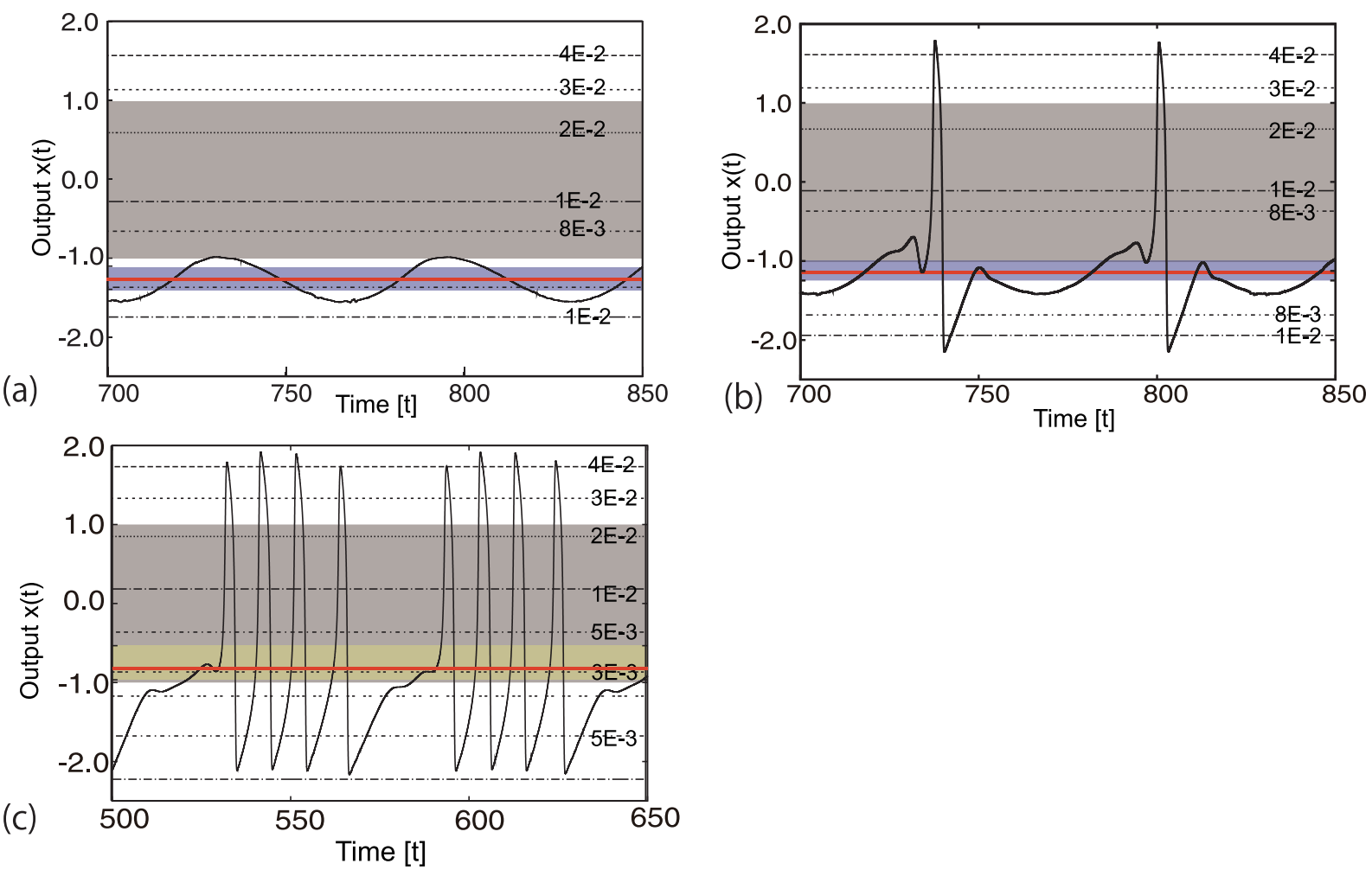

Fig. 8. Time series of the output, the potential, and the active areas of coupled van der Pol oscillators with $\epsilon_{x}=3.0, \epsilon_{y}=1.0, \beta_{x}=1.0, \beta_{y}=1.0, \theta_{y}=$ $0.0, \tau_{x}=1, \tau_{y}=100, \theta_{x}=-1.25$ (a), $\theta_{x}=-1.10(\mathrm{~b}), \theta_{x}=-0.80$ (c). Black lines, red lines, gray areas, yellow area, and blue areas denote the outputs $x(t)$, the equilibrium points, $b_{3}$ and $B_{1}$-active areas, $b_{1}$-active area, and $B_{2}$-active areas, respectively. 
$b_{2}(x)<0, b_{3}(x)<0, B_{1}(x)<0$, and $B_{2}(x)<0$ in the potential $U(x)$. Unlike in the case of single neuron model with three variables, the relation among active areas and an equilibrium point was complex compared to the single H-R model, because there are five active areas. However, we are able to find out a trend and discuss the external input-dependency of the output and the active areas. For example, $B_{2}$-active area described as a blue area always covers the equilibrium point when $\theta_{x}<-1$. When $-1<\theta_{x}$, the equilibrium point is covered by the $b_{1}$-active area described as a yellow active area. Taking another example, any one or both of the $b_{3}$ and $B_{1}$-active areas are situated in an area where $|x|<1$ is satisfied. This active area is described as a gray area in Fig. 8.

A quasi-particle takes the slow oscillating component by the $B_{2}$-active area in Fig. 8(a). The equilibrium point moves up increasing $\theta_{x}$ and this slow oscillation around equilibrium point moves in on the active area depicted as a gray area in Fig. 8(b). Then, a quasi-particle is provided the fast oscillating component by this active area and the output shows the spike pulse. Moreover increasing $\theta_{x}$, the equilibrium point moves up and the output has more spikes.

Considering the results in the case of single unit, we consider that the $B_{2}$ and $b_{1}$-active areas covering the equilibrium point occurs the slow oscillation from unit $y$. Further, the $b_{3}$ and $B_{1}$-active areas correspond to the active areas where unit $x$ has in stand-alone $\left[-\sqrt{\beta_{x}}<x<+\sqrt{\beta_{x}}\right]$, and they give the fast-oscillating component to a quasi-particle. The role of each active area is not reveal completely. However, the $B_{2}$ and $b_{1}$-active areas are found to cause the slow oscillation of the burst. Then, a quasi-particle moves in the $b_{3}$ and $B_{1}$-active areas, and the output shows some spikes the number of which per burst increases as the equilibrium point comes close and in these active areas.

\subsection{Fast-slow dynamics}

The coupled van der Pol oscillators shows different type of burst oscillation from the Hindmarsh-Rose model. The burst oscillation for the coupled van der Pol oscillators has the spike undershoot. Also, the burst dynamics has the external input-dependency of spike number. Now, therefore, we analyze this system in term of fast-slow coupled system in the similar way to the previous section, and discuss the behavior of unit $x$.

We treat Eqs. (31) and (32) as a fast-subsystem and a slow-subsystem, respectively. Focusing on unit $x$, we obtain the potential function $U(x, y)$ and an equilibrium point $x_{0}(y)$

$$
\begin{aligned}
& U(x, y)=-\frac{1}{\tau_{x}}\left\{\frac{1}{2} W x^{2}+\left(\omega y+\theta_{x}\right) x\right\}, \\
& x_{0}(y)=-\frac{\theta_{x}+\omega y}{W} .
\end{aligned}
$$

The potential function oscillates with time because of $y(t)$, and the stability of the equilibrium point varies too.

In the potential $U(x, y)$, there is only one active area where

$$
\epsilon_{x}\left(x^{2}-\beta_{x}\right)<0,
$$

is satisfied. We refer this active area as the $\xi$-active area. The $\xi$-active area $X_{\xi(x)<0}$ is calculated by Eq. (46)

$$
X_{\xi(x)<0} \equiv\left[-\sqrt{\beta_{x}}, \sqrt{\beta_{x}}\right] .
$$

Figures 9 and 10 show a bird's eye view of $U(x, y)$, a contour map, time series of the potential with active area, and the output $x(t)$. In this time, the equilibrium point $x_{0}(y)$ moves in and out of the $\xi$-active area in synchronization with output $y$. The equilibrium point goes into $X_{\xi(x)<0}$ at point (a) in Figs. 9 and 10, hence, the fast oscillation occurs. The equilibrium point is out of $X_{\xi(x)<0}$ after (b) in Figs. 9 and 10, and hence, the unit stays in a resting state again.

We consider that this slow oscillation of the potential by the input from unit $y$ corresponds to the slow oscillation around the $b_{1}$ and $B_{2}$-active areas. The particle takes the fast oscillating component from the $\xi$-active area. The $b_{3}$ and $B_{1}$-active areas occupy the same area as the $\xi$-active area does, hence, the fast oscillation also occurs around the $b_{3}$ and $B_{1}$-active areas. 


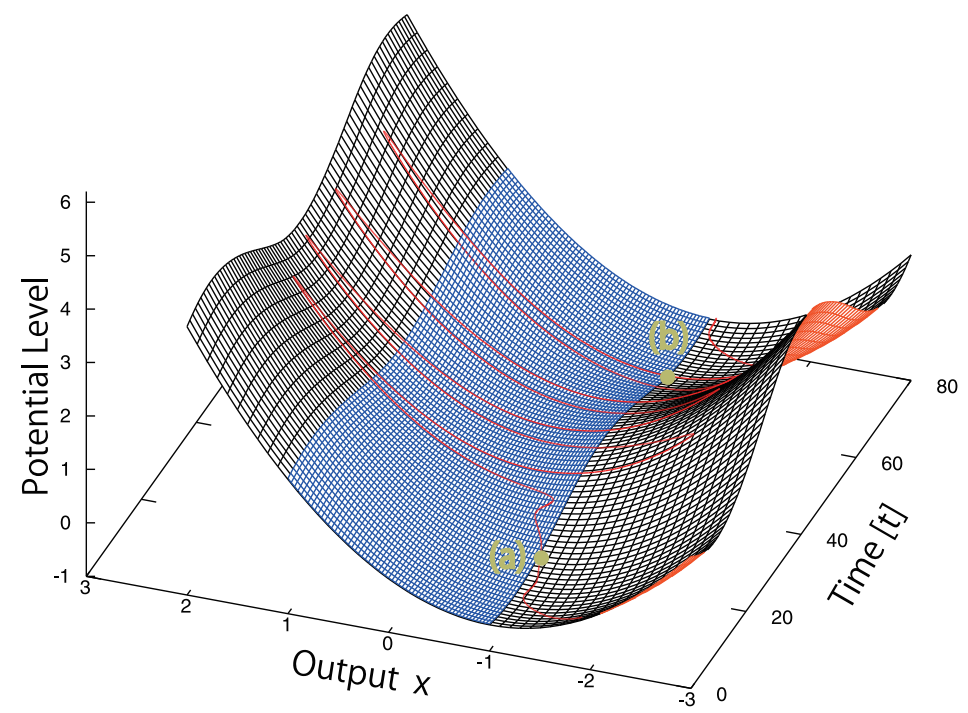

Fig. 9. Bird's eye view of time series of the output and the potential for the coupled van der Pol oscillator unit $x$. Blue zone and red line denote the $\xi$-active area and the solution orbit, respectively.

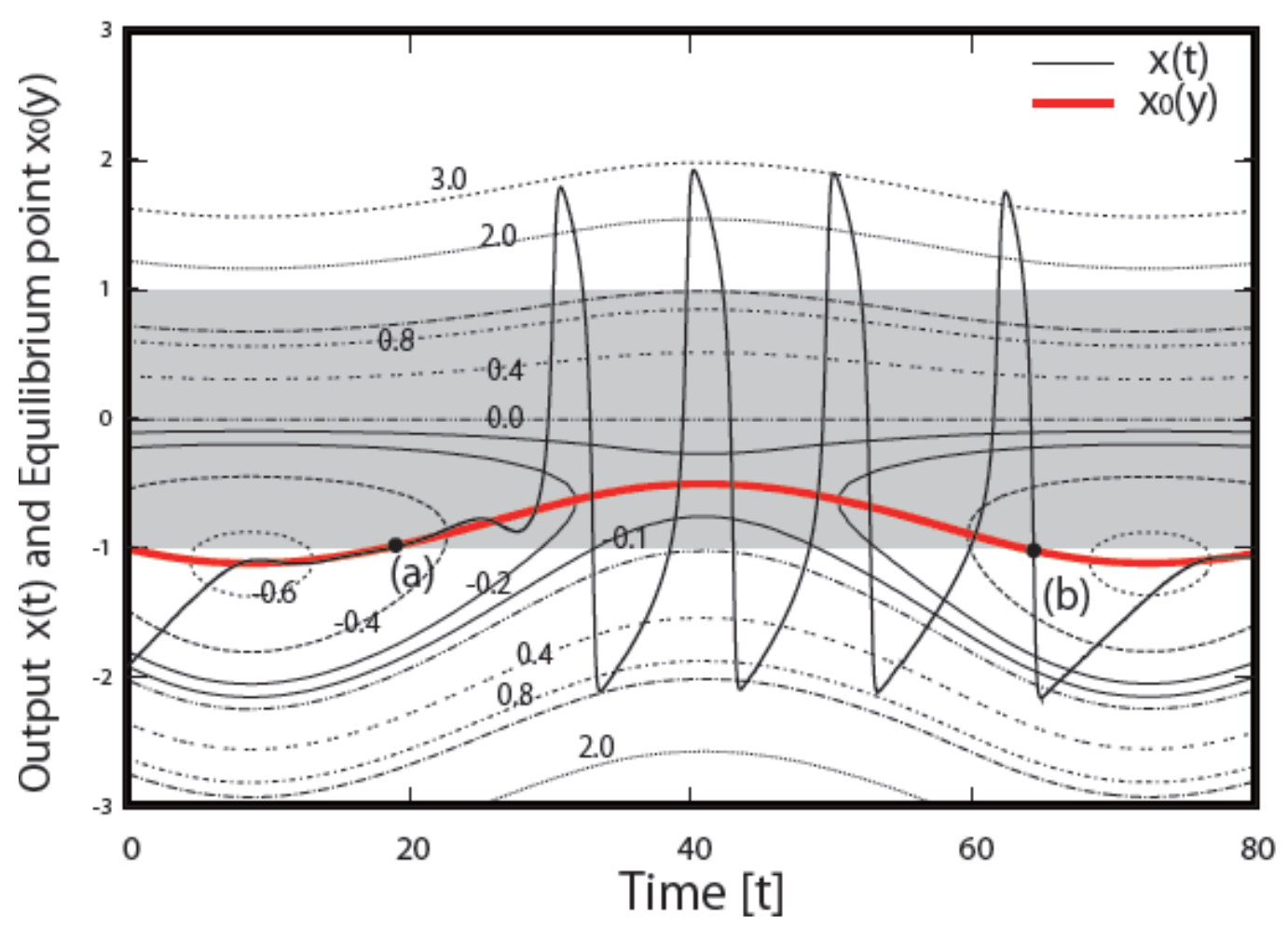

Fig. 10. Time series of the contour map of the potential for the coupled van der Pol oscillator unit. Black line, red line, and gray area denote the output $x(t)$, the trajectory of the equilibrium point $x_{0}(y)$ and the $\xi$-active area, respectively.

The firing oscillation and the resting state are occurred around the same equilibrium point, hence, the output shows the bursting pattern with spike undershoot. The number of spikes has close relation to the period during which the equilibrium point is in the $\xi$-active area. That is, it is easier for a particle to slow down as the equilibrium point is farther from $\xi$-active area, because a quasi particle loses its more energy by the damping except the inside of the active area. The external input $\theta_{x}$ defines the position of the equilibrium point, hence, the number of spikes depends on the amount of the external input $\theta_{x}$. 


\section{Conclusions}

In this paper we have provided an understanding of the burst firings with spatiotemporal constructions of the potential and the active areas, and claim that the active areas cause the eigen-oscillations individually. Hence, we dispose the active areas on the potential properly to design the intended wave forms. Then, the global curvature of the potential function ensures that these oscillations do not diverge.

We investigated two kinds of burst oscillations depending on the time constant $\tau_{x}$ for HindmarshRose model. It is the trigger of neuronal excitability that a stable and an unstable equilibrium point transmute into a stationary point. The dynamics occur with the stationary point's movement brought by the slow oscillation of variable $z$. Additionally, there is a relation between the slow oscillation of $z$ and the $b_{1}$-active area $X_{b_{1}(x)<0}$ in the one variable system. That is, $X_{b_{1}(x)<0}$ in one variable system corresponds to the slow oscillation of the potential $U(x, z)$ in the two variables system.

In contrast, the fast oscillations are dominated by the $\eta$-active areas in the two variables system. These active areas have relations to the amplitude of bursting spikes, hence, we are able to discuss the stability of the equilibrium point, and change the large amplitude vibration around the equilibrium point. In the one variable system, the fast oscillation is generated by the $b_{2}$-active area, and a change of the parameter $\tau_{x}$ affects the width of the interval between the $b_{1}$-active area and the $b_{2}$-active area, the width of the active area and the amplitude's characteristics of the bursting spikes. Therefore, the wider interval in (b) than (a) in Fig. 1 causes the tapering of the amplitude of fast oscillation, because a quasi particle loses more energy.

Additionally, we have applied our concept to the coupled van der Pol oscillators. And we achieved the burst oscillations and discussed the behavior. The burst oscillation with spike undershoot is observed when the equilibrium point is destabilized by the active area which dominates the slow oscillation, and the equilibrium point is situated near by the active areas which dominate the fast oscillation. It is the trigger of neuronal excitability that a stable equilibrium point moves into the $\xi$-active area in two variable system. The number of spikes has a close relation to the period during which the equilibrium point is in the $\xi$-active area, hence, we are able to control the number of spikes by varying the distance between the equilibrium point and active areas dominating the fast oscillation.

We claim that the active areas cause the eigen-oscillations individually, hence, we dispose the active areas on the potential properly to design the intended wave forms. The transition of the neuronal dynamics has a relation to the damping in the non-active areas around the equilibrium point and particularly ,the interval between the $b_{1}$ and the $b_{2}$-active areas. Then, the global curvature of the potential function ensures that these oscillations do not diverge. We consider that the method permits an implementation of the optional bursting dynamics for a neural network independent of neuron models.

Various bursting neuron models with higher order dimensions have been proposed including the Hindmarsh-Rose model. We consider that nonlinear equations of motion including the higher differential terms are explained through our technique, and we analyzed the coupled van der Pol system as preliminary steps for the networked systems. The generated behaviors of coupled two burst units will be studied in a forthcoming paper.

\section{Acknowledgments}

We would like to thank Dr. J. Sveholm for fruitful discussions.

\section{References}

[1] E.M. Izhikevich, "Neural excitability, spiking and bursting," International Journal of Bifurcation and Chaos, vol. 10, no. 6, pp. 1171-1266, 2006.

[2] I. Belykh, E. de Lange, and M. Hasler, "Synchronization of bursting neurons: What matters in the network topology," Phys. Rev. Letter., vol. 94, no. 188101, 2005.

[3] D. Somers and N. Kopell, "Waves and synchrony in networks of oscillators or relaxation and nonrelaxation type," Physica D89, pp. 169-183, 1995. 
[4] D. Terman and D. Wang, "Global competition and local cooperation in a network of neural oscillators," Physica D81, pp. 148-176, 1995.

[5] K. Nakajima and Y. Hayakawa, "Characteristics of inverse function delayed model for neural computation," Proc. NOLTA'02, pp. 861-864, October 2002.

[6] J.E. Lisman, "Bursts as a unit of neural information: Making unreliable synapses reliable," Trends in Neurosciences, vol. 20, no. 1, pp. 38-43, January 1997.

[7] E.M. Izhikevich, N.S. Desai and E.C. Hoppenstesdt, "Bursts as a unit of neural information: selective communication via resonance," Trends in Neurosciences, vol. 26, no. 3, pp. 161-167, March 2003.

[8] S. Suenaga, Y. Hayakawa, and K. Nakajima, "Design of a neural network chip for the burst ID model with ability of burst firing," IEICE Trans. Fundamentals, vol. E90-A, pp. 715-723, 2007.

[9] K. Nakajima and S. Suenaga, "Bursting characteristics of a neuron model based on a concept of potential with active areas," CHAOS 18, 023120, pp. 1-12, 2008.

[10] K. Kurose, Y. Hayakawa, and K. Nakajima, "Analyses of two dimensional hindmarsh-rose type model based-on a concept of potential with active areas," Proc. JNNS2009, pp. 108-109, Japan, September 2009.

[11] S. Tsuji, T. Ueta, H. Kawakami, H. Fujii and K. Aihara, "Bifurcations in two-dimensional hindmarsh-rose type model," International Journal of Bifurcation and Chaos, vol. 17, no. 3, pp. 985-998, 2007.

[12] J.L. Hindmarsh and R.M. Rose, "A model of neuronal bursting using three coupled first order differential equations," Proc. the Royal Society of London, Series B, Biological Sciences, vol. 221, pp. 87-102, 1984. 